\title{
Tetralogy of Fallot and the rubber heart model: Does the rubber meet the road?
}

\author{
William M. DeCampli, MD, PhD
}

\author{
From The University of Central Florida College of Medicine, Orlando, Fla; and The Heart Center at Arnold \\ Palmer Hospital for Children, Orlando, Fla. \\ Disclosures: Author has nothing to disclose with regard to commercial support. \\ Received for publication Oct 5, 2015; accepted for publication Oct 5, 2015; available ahead of print Nov 9, 2015. \\ Address for reprints: William M. DeCampli, MD, PhD, The Heart Center at Arnold Palmer Hospital for Children, \\ Pediatric Cardiothoracic Surgery, 50 W Sturtevant St, Orlando, FL 32806 (E-mail: william.decampli@ \\ orlandohealth.com). \\ J Thorac Cardiovasc Surg 2016;151:695-6 \\ $0022-5223 / \$ 36.00$ \\ Copyright (c) 2016 by The American Association for Thoracic Surgery \\ http://dx.doi.org/10.1016/j.jtcvs.2015.10.032
}

The preoperative predictors of salutary response to pulmonary valve replacement (PVR) in tetralogy of Fallot remain an enigma. Although numerous conventional factors have been considered, their calculated associations with objective outcome measures are inconsistent. ${ }^{1}$ In this issue of the Journal, Tang and coworkers ${ }^{2}$ present a computational dynamics (CD) study reporting an association between preoperative right ventricular (RV) wall stress (WS) and the change in ejection fraction $(\triangle E F)$ after PVR. They conclude that RV WS, calculated by $\mathrm{CD}$, is a potential tool to identify the response of RV function to PVR.

In this study, Tang and coworkers ${ }^{2}$ obtained preoperative values of instantaneous RV volume and pressure from magnetic resonance imaging and catheterization data, respectively. They then applied a parameterized model of RV tissue (the Mooney-Rivlin model relating WS to wall strain) to a 2-layer model of the RV. Because chamber pressure and volume are related to WS and wall strain, respectively, Tang and coworkers ${ }^{2}$ could determine the patient-specific parameter values of the Mooney-Rivlin equations by fitting them at the inner surface of the RV to the measured values at each time instant. They then calculated preoperative RV WS and wall strain at every point in time and location in the RV wall. Of several other variables calculated, they found that the maximum principal WS was the best single predictor of postoperative EF.

This study is a valuable contribution demonstrating the technique of integrating clinical data with computational models of the heart and circulation. Tang and coworkers have previously published innovative work on controlling RV strain in tetralogy of Fallot with RV modeling. ${ }^{3}$ The field is still evolving. For example, studies such as this typically use parameterized solid mechanical models (historically developed to describe rubberlike materials) validated with normal ventricular tissue. Such a model may not accurately represent the hypertrophied, dilated, fibrosed, remodeled RV in tetralogy of Fallot. Direct ex vivo mechanical measurements of the actual pathologic tissue are

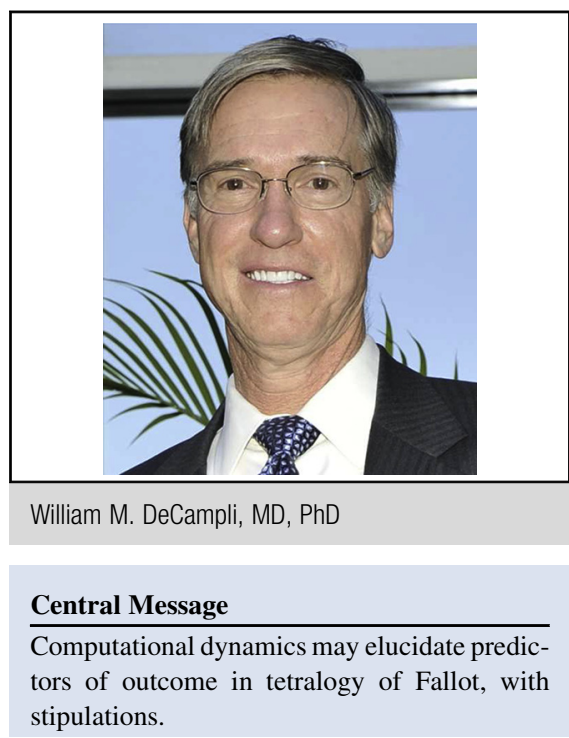

See Article page 687.

See Editorial page 623 .

preferable. In addition, computational studies sometimes omit important covariables that may influence the outcome. For example, the degree of RV outlet or pulmonary obstruction before and after the operation is not mentioned in this study of Tang and coworkers. ${ }^{2}$ Such obstruction directly affects EF and could affect the study results.

Assuming that the model is a reasonably good representation of the $\mathrm{RV}$, and that $\mathrm{RV}$ outlet or pulmonary obstruction was not repaired along with PVR in any of the patients, the calculated association between $\mathrm{WS}$ and $\Delta \mathrm{EF}$ is both statistically significant $\left(R^{2}=0.31 ; P=.026\right)$ and of potential clinical significance. There are 3 cautionary points to make here: (1) The result means that about only $31 \%$ of the variability in $\Delta E F$ can be explained by variations in preoperative WS. (2) The sample size is small. It can be shown that the addition of only 2 patients to the cohort (whose data are not "outliers") would render the calculated association between WS and $\Delta \mathrm{EF}$ insignificant. That is, with 16 patients, the calculated association is fragile. (3) Tang and coworkers ${ }^{2}$ place some emphasis on the calculated sensitivity $(80 \%)$ and specificity $(85 \%)$ of a "cutoff" value of stress (about $65 \mathrm{kPa}$ ), values above which predict poorer $\Delta \mathrm{EF}$ response. This latter calculation, however, is performed by condensing $\Delta \mathrm{EF}$ into a dichotomous variable so that almost all information about the variability 


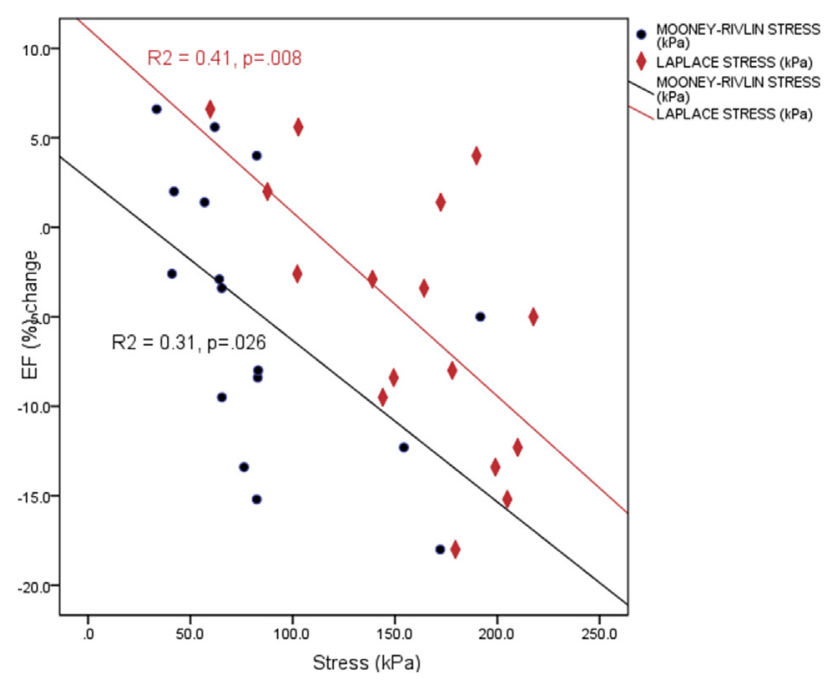

FIGURE 1. Computational dynamics in tetralogy of Fallot.

of $\Delta \mathrm{EF}$ is lost. The result is that the power to predict a "favorable" outcome can look good even when there is relatively weak correlation between WS and $\Delta \mathrm{EF}$, especially when the sample size is small. In the study of Tang and coworkers, ${ }^{2} 11$ of 16 patients actually had a negative $\triangle \mathrm{EF}$ after PVR, and the ones that improved did so by only an average of $5 \%$. The bottom line is that the clinician wants to know reliably how great $\Delta \mathrm{EF}$ is when WS has a certain value, and that is best established by a regression of $\triangle \mathrm{EF}$ as a continuous variable.

There is an additional interesting observation to make regarding the data presented. Given that the calculation of WS is complex and time-consuming, one may estimate it (without $\mathrm{CD}$ ) from a dimensional analog of the Laplace relation:

$$
\mathrm{WS}=\mathrm{c} \times \mathrm{P} \times \mathrm{V}^{1 / 3} / \mathrm{T}
$$

where $P, V$, and $T$ are the ventricular pressure, volume and wall thickness, respectively, as measured by magnetic resonance imaging and catheterization at the beginning of systole, and $c$ is an arbitrary scaling constant. The resulting regression of $\triangle \mathrm{EF}$ on WS is shown in Figure 1 (in red) compared with that of the more rigorously calculated Mooney-Rivlin stress (in black). The Mooney-Rivlin-based regression line is not better a fit to the patient data than the simple Laplace-based regression (for which $R^{2}=0.41$; $P=.008)$. This finding supports one concept: although $\mathrm{CD}$ can often give us very precise, detailed mechanical behavior with extraordinary spatial and time resolution, average, scalar, or "global" quantities, easy to measure, are sometimes sufficient to illustrate an association. In the present case, a larger sample size is necessary to determine whether, in the case of the rubber heart model, the rubber will meet the road.

\section{References}

1. Geva T. Indications for pulmonary valve replacement in repaired tetralogy of Fallot: the quest continues. Circulation. 2013;128:1855-7.

2. Tang D, Yang C, del Nido PJ, Zuo H, Rathod RH, Huang X, et al. Mechanical stress is associated with right ventricular response to pulmonary valve replacement in patients with repaired tetralogy of Fallot. J Thorac Cardiovasc Surg. 2016;151: 687-94.

3. Tang D, Yang C, Geva T, del Nido PJ. Right ventricular local longitudinal curvature as a marker and predictor for pulmonary valve replacement surgery outcome: an initial study based on preoperative and postoperative cardiac magnetic resonance data from patients with repaired tetralogy of Fallot. J Thorac Cardiovasc Surg. 2014;147:537-8. 